TRANSACTIONS OF THE

AMERICAN MATHEMATICAL SOCIETY

Volume 363, Number 4, April 2011, Pages 1925-1946

S 0002-9947(2010)05302-4

Article electronically published on November 16, 2010

\title{
ENERGY DISSIPATION AND SELF-SIMILAR SOLUTIONS FOR AN UNFORCED INVISCID DYADIC MODEL
}

\author{
D. BARBATO, F. FLANDOLI, AND F. MORANDIN
}

\begin{abstract}
A shell-type model of an inviscid fluid, previously considered in the literature, is investigated in absence of external force. Energy dissipation of positive solutions is proved, and decay of energy like $t^{-2}$ is established. Self-similar decaying positive solutions are introduced and proved to exist and classified. Coalescence and blow-up are obtained as a consequence, in the class of arbitrary sign solutions.
\end{abstract}

\section{INTRODUCTION}

The following system of differential equations,

$$
\begin{cases}X_{0}(t)=0 & \forall t \geq 0, \\ \dot{X}_{n}(t)=k_{n-1} X_{n-1}^{2}(t)-k_{n} X_{n}(t) X_{n+1}(t) & \forall t \geq 0, \quad \forall n \geq 1,\end{cases}
$$

where $k_{n}=2^{n}$, has been introduced as a simplified model of 3D Euler evolution in order to investigate a number of properties which are out of reach at present for more realistic models of fluid dynamics. Let us mention in particular the works 8 [11, [12, 5], 4, 6] and the references therein, devoted to this model and variants of it. This model differs from other dyadic or shell models, the analysis of which is more difficult and less explicit; see among many others [3, [10, [2, [7, 1]. System (1) has a basic monotonicity property that makes it more tractable.

Among the many interesting properties proved in the above mentioned works, let us recall: i) the dissipation of energy, in spite of the fact that formally the equation is conservative; ii) the blow-up of solutions in certain topologies, although the same solutions are global in larger spaces; iii) the relation with Kolmogorov (K41) scaling law. Our aim is to add some contribution to the understanding of these problems. The basic difference between the previous works dealing with energy dissipation and the present one is that a non-zero force was imposed until now, while we investigate the case without force, which contains a number of new phenomena.

Regarding energy dissipation, the results known until now have had the following form. A constant positive force $f>0$ is added to the first mode

$$
\dot{X}_{1}(t)=-k_{1} X_{1}(t) X_{2}(t)+f
$$

and it is proved that there exists a unique fixed point, having Kolmogorov scaling, which attracts exponentially all positive solutions (solutions with non-negative

Received by the editors October 30, 2008.

2010 Mathematics Subject Classification. Primary 35Q35, 76B03, 35Q30.

(C)2010 American Mathematical Society 
components), in the topology $l^{2}$. This implies that energy is dissipated. Positive solutions are better motivated in comparison with fluid dynamic equations (see [5]).

Although the case $f>0$ and the fixed point are very interesting, it is also of interest to analyze the free (unforced) dynamic, namely system (11) without any forcing term. Physically, if we accept that a dyadic model like (10) may describe something of turbulence, the unforced case would correspond to free decaying turbulence, a widely observed phenomenon; see 9] and the references therein. The results of energy dissipation from the previous papers do not extend to this case, they really require $f>0$ and make use of the non-trivial fixed point in the computations. For simplicity, one would conjecture the case $f=0$ to be similar (exponential decay to zero), maybe with a different proof, but it turns out this is not the case.

For the unforced case, namely system (1), we first prove the following result: all finite energy positive solutions have energy which decays to zero as $t \rightarrow \infty$. Nevertheless, for sufficiently regular initial conditions, energy is conserved for small times, as was shown for instance in [8], [1] and [14.

Even a large class of solutions starting with infinite energy immediately enter $l^{2}$. Namely, they immediately get finite energy and then continue their process of dissipation to zero.

However, the decay of energy to zero is no more exponential. By means of a scaling argument we prove an upper bound on the decay of the energy of the order $t^{-2}$. Then we prove a weak form of the lower bound of the same order, which includes in particular the property $\int_{0}^{\infty} X_{n}(t) d t=\infty$ for all $n$ greater than some $n_{0}$. Thus exponential decay is ruled out.

Then we investigate positive self-similar solutions of the form

$$
X_{n}(t)=\frac{a_{n}}{t-t_{0}}, \quad t \geq t_{0}
$$

We prove the existence of such solutions in the space $l^{2}$. The proof is highly non-trivial. The energy of these solutions decays exactly as $t^{-2}$, and we conjecture that this should be the case for all positive solutions.

The existence of such self-similar solutions also relates to the problem of blow-up and coalescence, but in a rather controversial way from the viewpoint of physical interest. If we decide that only solutions with positive components have physical meaning, our self-similar solutions do not contribute to the problem of blow-up. If on the contrary we consider system (11) as a non-linear model to be understood for any kind of initial condition, we have proved (by a simple inversion of time) that there exist solutions defined on the time interval $\left(-\infty, t_{0}\right)$ of the form

$$
X_{n}(t)=\frac{a_{n}}{t-t_{0}}, \quad t \leq t_{0}
$$

with $\left(a_{n}\right) \in l^{2}$. The components $X_{n}(t)$ are all negative. These solutions blow up in finite time, irrespective of the topology, in the sense that all components blow up in finite time. This result is much stronger than the blow-up results of the previous literature on dyadic models.

The existence of positive self-similar solutions also implies coalescence: we prove that at every point of a self-similar solution there is the coalescence of at least another solution (which cannot be positive). 
Let us finally mention other properties of the self-similar solutions we construct and some open problems.

Given $t_{0}$, we prove that there is a unique solution of the form (2) in $l^{2}$, with strictly positive components. We also prove that the components $a_{n}$ decay as

$$
a_{n} \sim C_{0} 2^{-n / 3}
$$

so, in a sense, these are 'Kolmogorov type decaying solutions' [13]. There are two degrees of freedom, however, in these solutions. One is the given value of $t_{0}$. The other is that we could take

$$
a_{1}=\ldots=a_{n_{0}}=0
$$

and prove that there exists a unique (for given $t_{0}$ ) self-similar solution with these first components equal to zero and all the others strictly positive. In this case, closer inspection gives us

$$
a_{n} \sim C_{n_{0}} 2^{-n / 3}
$$

where

$$
C_{n_{0}}=2^{-2 n_{0} / 3} R^{-1}, \quad R \in(4 / 5,1)
$$

(numerical experiments give us $R \approx 0.885765931$ ).

Thus the picture is that in $l^{2}$ there travels a family of self-similar solutions depending on the continuous parameter $t_{0}$ and the discrete parameter $n_{0}$.

What happens to all other solutions? Do they approach this set of special solutions? In this case, does a generic solution select one particular self-similar solution and get closer and closer to it, or does it slowly shift from one self-similar solution to the other? A sufficiently fast convergence to one self-similar solution would imply that Kolmogorov K41 scaling holds true for this simple dyadic model. But a slow convergence or a shift along different self-similar solutions could produce small deviations from Kolmogorov scaling. Further research is necessary to clarify these issues.

\section{Concepts of solution, their existence, Positivity AND ENERGY INEQUALITY}

To start, let us state rather general existence theorems. Their proofs are classical, compared to the previous literature, but at least the case of infinite initial energy is new, so we give the details in the Appendix. We denote by $H$ the space $l^{2}$, namely the space of all square integrable sequences of real numbers. It is a Hilbert space with the obvious inner product $\langle u, v\rangle_{H}=\sum_{n=1}^{\infty} u_{n} v_{n}$, where $u=\left(u_{n}\right)_{n \in \mathbb{N}}$, $v=\left(v_{n}\right)_{n \in \mathbb{N}}$, and $u, v \in H$. The corresponding norm in $H$ will be denoted by $|\cdot|_{H}$. We denote the space of all sequences of real numbers by $\mathbb{R}^{\mathbb{N}}$ and its subset of all non-negative sequences by $\mathbb{R}_{+}^{\mathbb{N}}$.

In the sequel we shall often use the term "energy" for the quantity $|X|_{H}^{2}$, for an element $X \in H$ or also $X \in \mathbb{R}^{\mathbb{N}}$ (it may have infinite energy).

Definition 1. Given $X^{0} \in \mathbb{R}^{\mathbb{N}}$, we call the componentwise solution of system (11) with initial condition $X^{0}$ any sequence $X=\left(X_{n}(\cdot)\right)_{n \in \mathbb{N}}$ of continuously differentiable functions $X_{n}(\cdot):[0, \infty) \rightarrow \mathbb{R}$ such that $X_{n}(0)=X_{n}^{0}$ for all $n \in \mathbb{N}$ and all equations in system (11) are satisfied. If $X(t) \in \mathbb{R}_{+}^{\mathbb{N}}$ for all $t \geq 0$, we call it a positive componentwise solution. 
If $X$ is a componentwise solution, from system (10) we have

$$
X_{n}(t)=X_{n}^{0} e^{-k_{n} \int_{0}^{t} X_{n+1}(r) d r}+\int_{0}^{t} e^{-k_{n} \int_{s}^{t} X_{n+1}(r) d r} k_{n-1} X_{n-1}^{2}(s) d s .
$$

This identity will be used quite often.

Definition 2. We say that a componentwise solution $X$ has finite energy for positive times if $X(t) \in H$ for all $t>0$. If also $X^{0} \in H$, we call $X$ a finite energy solution.

Theorem 3. Given $X^{0} \in \mathbb{R}_{+}^{\mathbb{N}}$, any componentwise solution of system (1) with initial condition $X^{0}$ is positive. At least one such solution exists. Moreover, any such solution has the following properties:

i) for every $n \geq 1$ and $t \geq 0$ we have

$$
\frac{d}{d t} \sum_{j=1}^{n} X_{j}^{2}(t)=-k_{n} X_{n}^{2}(t) X_{n+1}(t),
$$

and hence

$$
\sum_{j=1}^{n} X_{j}^{2}(t) \leq \sum_{j=1}^{n}\left(X_{j}^{0}\right)^{2}
$$

ii) if $X_{n}^{0}>0$ for some $n \geq 1$, then $X_{m}(t)>0$ for all $m \geq n$ and all $t>0$.

Theorem 4. For every $X^{0} \in H$, there exists at least one finite energy solution of system (11) with initial condition $X^{0}$, with the property

$$
|X(t)|_{H} \leq|X(s)|_{H} \quad \text { for all } \quad 0 \leq s \leq t .
$$

Moreover, if $X^{0} \in H \cap \mathbb{R}_{+}^{\mathbb{N}}$, then all componentwise solutions are finite energy and satisfy (5).

See the proofs in the Appendix.

\section{ENERGY DISSIPATION}

System (11) is formally conservative:

$$
\frac{1}{2} \frac{d}{d t}|X|_{H}^{2}=\sum_{n=1}^{\infty}\left(k_{n-1} X_{n} X_{n-1}^{2}-k_{n} X_{n}^{2} X_{n+1}\right)=0
$$

by a simple rearrangement of the series and the condition $X_{0}=0$. This rearrangement is rigorous if the solutions live in a sufficiently regular space (see for example [5]). Such a kind of regularity may hold for small times if the initial condition is very regular itself (see [8]), but for sufficiently large times we prove that solutions dissipate energy. The intuitive mechanism is a very fast shift of energy from small to large $n$ components.

We give two results of energy dissipation for positive solutions: infinite initial energy becomes finite immediately; the energy of a finite energy solution tends to zero as $t \rightarrow \infty$. Although the degree of infinity of the energy of initial conditions can be generalized, for the simplicity of statements we restrict ourselves to $X^{0}$ of class $l^{\infty}$ : the norm $\left\|X^{0}\right\|_{\infty}:=\sup _{n}\left|X_{n}^{0}\right|$ is finite. 
Theorem 5. Assume $X^{0} \in l^{\infty} \cap \mathbb{R}_{+}^{\mathbb{N}}$ and let $X$ be a positive componentwise solution of system (11) with initial condition $X^{0}$. Then $X$ has finite energy for positive times.

Theorem 6. If $X$ is a positive finite energy solution, then

$$
\lim _{t \rightarrow \infty}|X(t)|_{H}^{2}=0 \text {. }
$$

Moreover, given $L>0$ and $\alpha>0$, there exists $\bar{t}>0$ depending only on $L$ and $\alpha$ such that for all positive finite energy solutions $X$ with $|X(0)|_{H} \leq L$ we have $|X(\bar{t})|_{H}^{2} \leq \alpha$.

The proof of both statements is based on the following lemma.

Lemma 7. Let $X$ be a positive componentwise solution with $X(0) \in l^{\infty} \cap \mathbb{R}_{+}^{\mathbb{N}}$, and let $\|X(0)\|_{\infty} \leq L$. Let $\phi_{n}(t):=\sum_{k=1}^{n} X_{k}^{2}(t)$ for $n \geq 1$, and let $\phi_{0}(t) \equiv 0$ and $\phi_{\infty}(t):=\sum_{k=1}^{\infty} X_{k}^{2}(t)=|X(t)|_{H}^{2}$.

Then there exist two summable sequences of positive numbers $\left\{a_{n}\right\}_{n \geq 1}$ and $\left\{s_{n}\right\}_{n \geq 1}$ depending only on $L$, such that:

i) for all $n \geq 1$, for all $t>0$ and for all $\varepsilon \in(0,1]$ one has

$$
\phi_{n}\left(t+\varepsilon^{-2} s_{n}\right)-\phi_{n-1}(t) \leq \varepsilon a_{n} ;
$$

ii) for all integers $M \geq 1$ one has

$$
\phi_{\infty}\left(\varepsilon^{-2} \sum_{k=M}^{\infty} s_{k}\right) \leq \phi_{M-1}(0)+\varepsilon \sum_{n=M}^{\infty} a_{n} .
$$

Proof. For all $n$, let $m_{n}^{2}:=\phi_{n}(0)$, so that by equation (4), $X_{n}(t) \leq m_{n}$ for all $t \geq 0$.

We observe that it is possible to find two summable sequences $\left\{a_{n}\right\}_{n}$ and $\left\{s_{n}\right\}_{n}$ such that for all $n \geq 1$,

$$
2^{n} s_{n} a_{n}^{2} \geq 2 m_{n}^{2} m_{n+2}\left(1+\frac{1}{2^{n} s_{n} m_{n+2}}\right) .
$$

It is enough to set $s_{n}=2^{-n / 4}$ and $a_{n}=C 2^{-n / 4}$ with $C$ a suitable constant, and recall that by hypothesis $m_{n} \leq L \sqrt{n}$. We observe that without changing the sequences, a fortiori for all $\varepsilon \leq 1$,

$$
2^{n} s_{n} a_{n}^{2} \geq 2 m_{n}^{2} m_{n+2}\left(1+\frac{1}{\varepsilon^{-2} 2^{n} s_{n} m_{n+2}}\right) .
$$

Part 1. Given the two sequences, we now show that the upper bounds (6) hold. Let $h=\varepsilon^{-2} s_{n}$. Since $\phi$ is non-increasing, for all $s \in[0, h]$,

$$
\phi_{n}(t+h)-\phi_{n-1}(t) \leq \phi_{n}(t+s)-\phi_{n-1}(t+s)=X_{n}^{2}(t+s) .
$$

Hence, if for some $s$ inside the interval, $X_{n}^{2}(t+s) \leq \varepsilon a_{n}$, we are done.

On the other hand, let us suppose that $X_{n}^{2}(t+s)>\varepsilon a_{n}$ for all $0 \leq s \leq h$. One has

$$
\phi_{n}(t+h)-\phi_{n-1}(t)=X_{n}^{2}(t)+\int_{0}^{h} \frac{d}{d s} \phi_{n}(t+s) d s .
$$

We need to prove that

$$
\int_{0}^{h} \frac{d}{d s} \phi_{n}(t+s) d s \leq \varepsilon a_{n}-X_{n}^{2}(t) .
$$


For sake of notational simplicity, let $a=\varepsilon a_{n} k_{n}$ and let $\lambda=k_{n+1} m_{n+2}$. Then, by Theorem 3(i),

$$
\int_{0}^{h} \frac{d}{d s} \phi_{n}(t+s) d s=\int_{0}^{h}-k_{n} X_{n}^{2}(t+s) X_{n+1}(t+s) d s \leq-a \int_{0}^{h} X_{n+1}(t+s) d s .
$$

We need a lower bound for $X_{n+1}$. In the interval $[t ; t+h]$ we know that

$$
\dot{X}_{n+1}=k_{n} X_{n}^{2}-k_{n+1} X_{n+1} X_{n+2} \geq a-\lambda X_{n+1},
$$

whence we get

$$
X_{n+1}(t+s) \geq e^{-\lambda s} X_{n+1}(t)+\frac{a}{\lambda}\left(1-e^{-\lambda s}\right) \geq \frac{a}{\lambda}\left(1-e^{-\lambda s}\right) .
$$

By substituting into the integral above one gets

$$
\int_{0}^{h} \frac{d}{d s} \phi_{n}(t+s) d s \leq-\frac{a^{2}}{\lambda} \int_{0}^{h}\left(1-e^{-\lambda s}\right) d s=-\frac{a^{2}}{\lambda^{2}}\left(e^{-\lambda h}-1+\lambda h\right) \leq-\frac{a^{2}}{\lambda^{2}} \frac{\lambda h}{1+\frac{2}{\lambda h}} .
$$

By next substituting $h, a$ and $\lambda$ and recalling condition (8), we finally get

$$
\begin{aligned}
\int_{0}^{h} \frac{d}{d s} \phi_{n}(t+s) d s & \leq-\frac{a^{2} h}{\lambda\left(1+\frac{2}{\lambda h}\right)}=-\frac{2^{n} s_{n} a_{n}^{2}}{2 m_{n+2}\left(1+\frac{1}{\varepsilon^{-2} 2^{n} s_{n} m_{n+2}}\right)} \\
& \leq-m_{n}^{2} \leq \varepsilon a_{n}-X_{n}^{2}(t) .
\end{aligned}
$$

This concludes the first part.

Part 2. Let $M \geq 1$ and define the sequence $\left\{t_{n}\right\}_{n \geq M-1}$ by $t_{M-1}=0$ and $t_{n}=\varepsilon^{-2} \sum_{k=M}^{n} s_{k}$, for $n \geq M$. We substitute $t=t_{n-1}$ inside the inequality (6):

$$
\phi_{n}\left(t_{n}\right)-\phi_{n-1}\left(t_{n-1}\right)=\phi_{n}\left(t_{n-1}+\varepsilon^{-2} s_{n}\right)-\phi_{n-1}\left(t_{n-1}\right) \leq \varepsilon a_{n} .
$$

Adding the above inequalities for $n$ from $M$ to any number $N>M$, one has

$$
\phi_{N}\left(t_{N}\right)-\phi_{M-1}(0)=\sum_{n=M}^{N}\left[\phi_{n}\left(t_{n}\right)-\phi_{n-1}\left(t_{n-1}\right)\right] \leq \varepsilon \sum_{n=M}^{N} a_{n} .
$$

Monotonicity of $\phi_{N}$ yields that

$$
\phi_{N}\left(\varepsilon^{-2} \sum_{k=M}^{\infty} s_{k}\right) \leq \phi_{N}\left(t_{N}\right) \leq \phi_{M-1}(0)+\varepsilon \sum_{n=M}^{N} a_{n} ;
$$

hence letting $N$ go to infinity we get (77). The proof of the lemma is complete.

We are now ready to prove the above theorems. Consider the assumptions of Theorem [5. By letting $\varepsilon=1$ in the second part of Lemma 7 one has that $X(t) \in H$ for $t \geq \sum_{k=M}^{\infty} s_{k}$. Letting $M$ go to infinity we get the thesis of Theorem 5 ,

As for Theorem [6. let us prove that for all $\alpha>0$ there exists some $\bar{t}>0$ such that $\phi_{\infty}(\bar{t}) \leq \alpha$. Since $\|X(0)\|_{\infty} \leq|X(0)|_{H} \leq L$, we let $M=1$ in the second part of Lemma 7; we only need to choose $\varepsilon$ in such a way that $\varepsilon \sum_{n=1}^{\infty} a_{n} \leq \alpha$. One gets $\bar{t}=\varepsilon^{-2} \sum_{k=1}^{\infty} s_{k}$. The proof is complete. 


\section{Bounds ON THE DECAY OF ENERGY AS $t \rightarrow \infty$}

In this section we prove a bound from above and another from below, for the decay of energy as $t \rightarrow \infty$, which essentially says that solutions decay as $t^{-1}$. The results are restricted to positive componentwise solutions. The first result is due to a scaling argument based on the fact that the non-linearity is homogeneous of degree two.

Theorem 8. Let $X$ be a positive componentwise solution with $X(0) \in l^{\infty} \cap \mathbb{R}_{+}^{\mathbb{N}}$. Then there exists $C>0$ such that

$$
|X(t)|_{H}^{2} \leq \frac{C}{t^{2}}
$$

for $t \geq 1$.

Proof. First, by Theorem [5] the solution has finite energy for positive times. Hence, by Theorem $\left[\right.$, there is a time $t_{0}$ such that $\left|X\left(t_{0}\right)\right|_{H}^{2} \leq 1$. Thus, it is not restrictive to prove the theorem in the case $\left|X^{0}\right|_{H}^{2}=1$.

First a general fact: from Theorem [6] we know that there exists $\bar{t}>0$ such that for all initial conditions $X^{0}$ with $\left|X^{0}\right|_{H}^{2}=1$ we have

$$
|X(\bar{t})|_{H}^{2} \leq \frac{1}{4}
$$

Let us start the proof for a solution $X$ such that $\left|X^{0}\right|_{H}^{2}=1$. Let $\alpha_{1}^{2}=|X(\bar{t})|_{H}^{2}$. Consider the rescaled $H$-valued function $Y=\left(Y_{n}\right)_{n \in \mathbb{N}}$ defined as

$$
Y(t):=\alpha_{1}^{-1} X\left(\alpha_{1}^{-1} t+\bar{t}\right) .
$$

We have

$$
\begin{gathered}
\dot{Y}_{n}(t)=\alpha_{1}^{-2} k_{n-1} X_{n-1}^{2}\left(\alpha_{1}^{-1} t+\bar{t}\right)-\alpha_{1}^{-2} k_{n} X_{n}\left(\alpha_{1}^{-1} t+\bar{t}\right) X_{n+1}\left(\alpha_{1}^{-1} t+\bar{t}\right) \\
=k_{n-1} Y_{n-1}^{2}(t)-k_{n} Y_{n}(t) Y_{n+1}(t) .
\end{gathered}
$$

This means that $Y$ is a finite energy solution of system (10). Moreover, $|Y(0)|_{H}^{2}=1$. Hence

In terms of $X$ we have

$$
\alpha_{2}^{2}:=|Y(\bar{t})|_{H}^{2} \leq \frac{1}{4}
$$

$$
\left|X\left(\frac{\bar{t}}{\alpha_{1}}+\bar{t}\right)\right|_{H}^{2}=\alpha_{1}^{2} \alpha_{2}^{2}
$$

By induction we can prove that

$$
\left|X\left(\frac{\bar{t}}{\alpha_{1} \cdot \alpha_{2} \cdot \ldots \cdot \alpha_{k}}+\cdots+\frac{\bar{t}}{\alpha_{1}}+\bar{t}\right)\right|_{H}^{2}=\alpha_{1}^{2} \cdot \alpha_{2}^{2} \cdot \ldots \cdot \alpha_{k+1}^{2}
$$

for every $k \geq 1$. Since $\alpha_{i} \leq \frac{1}{2}$, we have

$$
\frac{\bar{t}}{\alpha_{1} \cdot \alpha_{2} \cdot \ldots \cdot \alpha_{k}}+\cdots+\frac{\bar{t}}{\alpha_{1}}+\bar{t}<\frac{2 \bar{t}}{\alpha_{1} \cdot \alpha_{2} \cdot \ldots \cdot \alpha_{k}} \text {. }
$$

Recall that energy inequality holds for all positive finite energy solutions; see Theorem 3. Hence for all $t \geq \frac{2 \bar{t}}{\alpha_{1} \cdot \alpha_{2} \cdot \ldots \cdot \alpha_{k}}$ we have

$$
|X(t)|_{H}^{2} \leq \alpha_{1}^{2} \cdot \alpha_{2}^{2} \cdot \ldots \cdot \alpha_{k+1}^{2} \text {. }
$$


If we restrict ourselves to $\frac{2 \bar{t}}{\alpha_{1} \cdot \alpha_{2} \cdot \ldots \cdot \alpha_{k}} \leq t \leq \frac{2 \bar{t}}{\alpha_{1} \cdot \alpha_{2} \cdot \ldots \cdot \alpha_{k} \cdot \alpha_{k+1}}$ we also have $\alpha_{1}^{2} \cdot \alpha_{2}^{2} \cdot \ldots$. $\alpha_{k+1}^{2} \leq \frac{4 \bar{t}^{2}}{t^{2}}$. Hence

$$
|X(t)|_{H}^{2} \leq \frac{4 \bar{t}^{2}}{t^{2}}
$$

for all $\frac{2 \bar{t}}{\alpha_{1} \cdot \alpha_{2} \cdot \ldots \cdot \alpha_{k}} \leq t \leq \frac{2 \bar{t}}{\alpha_{1} \cdot \alpha_{2} \cdot \ldots \cdot \alpha_{k} \cdot \alpha_{k+1}}$. This implies the claim of the theorem. The proof is complete.

Theorem 9. Let $X$ be a positive componentwise solution with $X(0) \in l^{\infty} \cap \mathbb{R}_{+}^{\mathbb{N}}$. Let $n_{0}+1$ be the minimum integer with the property $X_{n_{0}+1}^{0}>0$. We know from Theorem 3 that $X_{n}(t)>0$ for all $n>n_{0}$ and $t>0$. Then, for some constant $C>0$, for every $n>n_{0}$ and $t \geq 1$ we have

$$
\int_{1}^{t} X_{n+1}(s) d s \geq k_{n}^{-1} \log t+k_{n}^{-1} \log \left(\frac{X_{n}(1)}{C}\right) .
$$

Thus, in particular, for every $n>n_{0}$,

$$
\int_{1}^{\infty} X_{n+1}(s) d s=\infty
$$

and

$$
\limsup _{t \rightarrow \infty} t \cdot X_{n+1}(t) \geq k_{n}^{-1} .
$$

Proof. From identity (3) we have

$$
X_{n}(t) \geq X_{n}(1) e^{-k_{n} \int_{1}^{t} X_{n+1}(s) d s} .
$$

By the upper bound of the previous theorem, there exists a constant $C>0$ such that

Hence

$$
X_{n}(1) e^{-k_{n} \int_{1}^{t} X_{n+1}(s) d s} \leq X_{n}(t) \leq \frac{C}{t}
$$

$$
-k_{n} \int_{1}^{t} X_{n+1}(s) d s \leq \log \left(\frac{C}{X_{n}(1) \cdot t}\right)
$$

namely

$$
\int_{1}^{t} X_{n+1}(s) d s \geq \frac{\log \left(\frac{X_{n}(1)}{C}\right)+\log t}{k_{n}} .
$$

This implies the claims of the theorem. The proof is complete.

\section{Self-Similar SOlutions AND RElated FaCtS}

We call self-similar any solution $X$ of the form $X_{n}(t)=a_{n} \cdot \varphi(t)$. It is easy to verify that self-similar solutions satisfying the equations in (1) have the form

$$
X_{n}(t)=\frac{a_{n}}{t-t_{0}}, \quad t>t_{0}
$$

with $t_{0}<0$. We are interested in finite energy self-similar solutions; hence we also require

$$
\sum_{n=1}^{\infty} a_{n}^{2}<\infty
$$

In the next section we prove the following result. 
Theorem 10. Given $t_{0}<0$, there exists a unique finite energy self-similar solution with $a_{1} \neq 0$. In general, given $t_{0}<0$ and $n_{0} \geq 0$, there exists a unique finite energy self-similar solution with

$$
a_{1}=\ldots=a_{n_{0}}=0, \quad a_{n_{0}+1} \neq 0
$$

(where the first conditions are meaningful only for $n_{0}>0$ ). In addition, the coefficients $a_{n}$ have the property

$$
\lim _{n \rightarrow \infty} \frac{a_{n}}{k_{n}^{-1 / 3}}=C_{n_{0}} .
$$

Thus we see that Kolmogorov scaling law [13] (so-called K41) appears in these special solutions, phenomenologically associated to decaying turbulence. But it is a very difficult open problem for us to understand whether all other solutions approach the self-similar ones and in which sense, so we cannot say how general this scaling property should be considered.

The existence of finite energy self-similar solutions is of conceptual interest in itself, in comparison with analogous investigations for Euler and Navier-Stokes equations. Also here, in this very simple context, the proof is highly non-trivial. Apart from its intrinsic interest, the existence of such solutions has a number of implications.

First, they realize perfectly the decay $t^{-1}$, coherently with the previous section (where the lower bound was more vague). We conjecture that the set of all finite energy self-similar solutions, set depending on $t_{0} \in \mathbb{R}$ and $n_{0} \geq 0$, attracts all other solutions in a suitable sense. If this is the case, the decay $t^{-1}$ would be more strictly the true one for all solutions.

A second implication is the existence of solutions that blow up backward in time. This is of interest for two reasons. To explain them let us first clarify what happens to solutions when we reverse time.

We may consider system (11) for negative times, $t \leq 0$, and give a definition of componentwise solution exactly as for $t \geq 0$. All definitions and theorems can be rewritten for negative times. One also has the following correspondence between the forward and backward problem: let $X=(X(t))_{t \geq 0}$ be a componentwise solution of system (1), for $t \geq 0$, as usual. Then $Y=(Y(t))_{t \leq 0}$ defined as

$$
Y(t)=-X(-t)
$$

is a componentwise solution for $t \leq 0$. Indeed, by the purely quadratic nature of the equation,

$$
\begin{aligned}
\dot{Y}_{n}(t) & =\dot{X}_{n}(-t)=k_{n-1} X_{n-1}^{2}(-t)-k_{n} X_{n}(-t) X_{n+1}(-t) \\
& =k_{n-1} Y_{n-1}^{2}(t)-k_{n} Y_{n}(t) Y_{n+1}(t) .
\end{aligned}
$$

Thus, any positive solution over $[0, \infty)$ gives rise to a negative solution over $(-\infty, 0]$, and vice versa.

Theorem 10 ensures that there exists a self-similar solution $X_{n}(t):=\frac{a_{n}}{t-t_{0}}$ with $t_{0}<0$ and $a_{n}>0$ for all $n>n_{0}$. It is easy to check that $X_{n}(t)$ is a componentwise solution on the open interval $\left(t_{0},+\infty\right)$. The energy is finite for all $t>t_{0}$ and $\lim _{t \rightarrow \infty}\left|X_{n}(t)\right|=0$, whereas $\lim _{t \rightarrow t_{0}^{+}} X_{n}(t)=+\infty$ for all $n>n_{0}$. With time inversion (10) it is possible to define $Y(t):=-X(-t)$ for all $n$ and $t \in\left(-\infty,-t_{0}\right) . \quad Y(t)$ is a componentwise solution of (11) on the open interval $\left(-\infty,-t_{0}\right)$ and $\lim _{t \rightarrow-\infty}\left|Y_{n}(t)\right|=0$, whereas $\lim _{t \rightarrow-t_{0}^{-}}\left|Y_{n}(t)\right|=+\infty$. This means 
that $Y_{n}$ blows up in finite time. Notice that every component blows up, not only some norm of the solution.

A consequence of this is a coalescence property of self-similar solutions. Theorem 4 applied with initial condition $Y(0)$ states that there exists a finite energy solution $\tilde{Y}(t)$, defined for all $t \geq 0$, with bounded energy and initial condition $\tilde{Y}(0)=Y(0)$. Moreover, condition (5) of that theorem ensures that the energy of $\tilde{Y}$ is non-increasing in $t$, on the contrary $|Y|_{H}$ is increasing in time. This means that on the system (1) there is not uniqueness of solutions in $\mathbb{R}^{\mathbb{N}}$. This result is of interest in itself, but we stress that it holds in the enlarged class of non-necessarily positive solutions.

Let us invert the time again with $\tilde{X}_{N}(t):=-\tilde{Y}_{N}(-t) . X$ and $\tilde{X}$ are two componentwise solutions on the interval $\left(t_{0}, 0\right]$, with decreasing in time and non-decreasing in time energy, respectively. They are different on $\left(t_{0}, 0\right)$ and coincide at time $t=0$. The role of time $t=0$ in the previous argument can be replaced by any time $t_{1}>t_{0}$. Thus we have

Corollary 11. If $X$ is a self-similar solution of the form (91), all its values are coalescence points in the sense that for all $t_{1} \geq t_{0}$ there exists a finite energy solution $X^{t_{1}}$, defined for $t \in\left(-\infty, t_{1}\right]$, such that $X^{t_{1}}\left(t_{1}\right)=X\left(t_{1}\right)$ and $X^{t_{1}}(t) \neq X(t)$ on $\left(t_{0}, t_{1}\right)$.

Finally, we state the blow-up for negative self-similar finite energy solutions.

Corollary 12. Given $t_{0}>0$ and $n_{0} \geq 0$, there exists a unique negative finite energy self-similar solution, defined on $\left[0, t_{0}\right)$ with

$$
a_{1}=\ldots=a_{n_{0}}=0, \quad a_{n_{0}+1} \neq 0 .
$$

This solution blows up at time $t_{0}$.

Proof. Apply the inversion (10) to the solution given by Theorem 10 .

\section{Existence AND UNIQUENESS OF SELF-SIMILAR SOLUTIONS}

In order to prove Theorem 10 it is best to restate the problem in terms of properties for sequences of positive numbers.

If a positive componentwise solution is of the form (9), then

$$
\begin{aligned}
-\frac{a_{n}}{\left(t-t_{0}\right)^{2}}=\dot{X}_{n}(t) & =k_{n-1} X_{n-1}^{2}(t)-k_{n} X_{n}(t) X_{n+1}(t) \\
& =k_{n-1} \frac{a_{n-1}^{2}}{\left(t-t_{0}\right)^{2}}-k_{n} \frac{a_{n} a_{n+1}}{\left(t-t_{0}\right)^{2}},
\end{aligned}
$$

so the sequence $\left\{a_{n}\right\}$ must satisfy

$$
a_{n} a_{n+1}=2^{-n} a_{n}+a_{n-1}^{2} / 2
$$

for all $n$. It is indeed possible for the first terms $a_{1}, a_{2}, \ldots, a_{n_{0}}$ to be zero, but by induction, if $a_{n_{0}+1}>0$, then the subsequent coefficients must satisfy

$$
a_{n+1}=2^{-n}+\frac{a_{n-1}^{2}}{2 a_{n}}>0, \quad n \geq n_{0}+1 .
$$

To simplify matters and take into account $n_{0}$, let

$$
\widetilde{a}_{n}:=2^{n+n_{0}} a_{n+n_{0}}
$$


so that the condition becomes $\widetilde{a}_{0}=0, \widetilde{a}_{1}>0$ and

$$
\widetilde{a}_{n+1}=2+4 \frac{\widetilde{a}_{n-1}^{2}}{\widetilde{a}_{n}}, \quad n \geq 1 .
$$

Since, given $a_{n_{0}+1}$ or $\widetilde{a}_{1}$, this recurrence uniquely defines the sequence, in order to prove Theorem 10 we have to show that there exists a unique positive number $\widetilde{a}_{1}$ such that the sequence $\left\{a_{n}\right\}_{n} \in H$. We will prove the following.

Theorem 13. There exists a unique real number $\gamma$ such that the sequence $\left\{a_{n}\right\}_{n}$ is in $H$ iff $\widetilde{a}_{1}=\gamma$ (equivalently, iff $a_{0}=a_{1}=\cdots=a_{n_{0}}=0$ and $a_{n_{0}+1}=2^{-n_{0}-1} \gamma$ ).

Moreover, let $\beta=2^{-1 / 3}$. One can find $R>0$ and a strictly decreasing bijective function $h:(0 ; R] \rightarrow[0 ; \infty)$ such that

i) $\gamma=h\left(\beta^{2} R\right)$,

ii) if $\left\{a_{n}\right\}_{n} \in H$, then $a_{n}=2^{-n} h\left(\beta^{2\left(n-n_{0}\right)} R\right)$ for all $n>n_{0}$. In particular $a_{n} \sim C_{n_{0}} \beta^{n}$, for $n \rightarrow \infty$, with $C_{n_{0}}=\beta^{2 n_{0}} / R$.

Numerical computations give $\gamma \approx 0.917576296$.

It is clear that Theorem 10 follows immediately from Theorem 13. The proof of the latter requires some work, and will follow from Theorem 17 below. Three technical lemmas will be needed.

We start by looking for another sequence $\left\{d_{k}\right\}_{k \geq-1}$ (not depending on $a_{n}$ ) satisfying the peculiar relation below:

$$
\left\{\begin{array}{l}
d_{-1}=-1, \\
\sum_{i=-1}^{k+1} d_{i} d_{k-i}\left(4-\beta^{2 k+2 i}\right)=2 d_{k} \beta^{2 k}, \quad k \geq-1,
\end{array}\right.
$$

Lemma 14. The equations (13) uniquely define a sequence of real numbers $\left\{d_{k}\right\}_{k \geq-1}$ such that $d_{k} \geq 0$ for $k \geq 0$. The power series $\sum_{k=0}^{\infty} d_{k} x^{k}$ has a positive convergence radius $R>0$. If we let $h(x):=-\sum_{k=-1}^{\infty} d_{k} x^{k}$, the function $h$ is defined on the interval $(0 ; R)$ where it is analytic, non-negative and strictly decreasing, with $h\left(0^{+}\right)=+\infty$ and $h\left(R^{-}\right)=0$ (so that it can be continuously extended on $(0 ; R])$.

Proof. The second part of (13) uniquely defines $d_{k+1}$ as a function of the previous terms. Truly, the coefficient of $d_{k+1}$ is $d_{-1}\left(8-\beta^{2 k-2}-\beta^{4 k+2}\right)$, which is always non-zero.

With some algebraic manipulations, the above system of equations can be rewritten as

$$
\left\{\begin{array}{l}
d_{-1}=-1 \\
d_{0}=\left(2 \beta^{-1}-\beta-\beta^{3}\right)^{-1} \approx 0.8155665, \\
d_{k+1}=\frac{1}{2} \sum_{i=0}^{k} \alpha_{k, i} d_{i} d_{k-i},
\end{array} \quad k \geq 0\right.
$$

where

$$
\alpha_{k, i}= \begin{cases}\frac{1+\beta^{4}-\beta^{-1}}{1-\beta^{7}-\beta^{11}}, & k=0, \quad i=0, \\ \frac{\left(1-\beta^{2 k+2}\right)\left(1+\beta^{2 k+7}\right)}{1-\beta^{2 k+7}-\beta^{4 k+11}}, & k>0, \quad i=0, k, \\ \frac{1-\beta^{4 k-2 i+9}-\beta^{2 k+2 i+9}}{1-\beta^{2 k+7}-\beta^{4 k+11}}, & 0<i<k .\end{cases}
$$


All $\alpha_{k, i}$ are positive, and so $d_{k} \geq 0$ for $k \geq 0$. We notice moreover that $\lim _{k \rightarrow \infty} \alpha_{k, i}$ $=1$ uniformly in $i$ (exponentially in $k$; see the Appendix).

The recursion (14) is similar to the classical Catalan sequence, but since we have only some asymptotic control on the coefficients $\alpha_{k, i}$, and since the behaviour of $\left\{d_{k}\right\}_{k}$ strongly depends on the first values (even its convergence radius does), we need some explicit investigation of its properties.

For $k \geq 0$, let $d_{k+1}^{\prime}:=\frac{1}{2} \sum_{i=0}^{k}\left(\alpha_{k, i}-1\right) d_{i} d_{k-i}$ and let $d_{0}^{\prime}=d_{0}$.

Let $g(x):=\sum_{k=0}^{\infty} d_{k} x^{k}$ so that $h(x)=x^{-1}-g(x)$. Let $\hat{g}(x):=\sum_{k=0}^{\infty} d_{k}^{\prime} x^{k}$. By the third part of (14) it follows that

$$
\sum_{k=n}^{\infty} d_{k+1} x^{k+1}=x \frac{1}{2} \sum_{k=n}^{\infty} \sum_{i=0}^{k} \alpha_{k, i} d_{i} x^{i} d_{k-i} x^{k-i} .
$$

If $n$ is such that $\left|\alpha_{k, i}-1\right|<\epsilon$, we have

$$
g(x)-\sum_{k=0}^{n} d_{k}^{\prime} x^{k} \leq x \frac{1+\epsilon}{2} \sum_{k=0}^{\infty} \sum_{i=0}^{k} d_{i} x^{i} d_{k-i} x^{k-i}=\frac{1+\epsilon}{2} x g^{2}(x) .
$$

Also writing the corresponding lower bound and letting $\epsilon \rightarrow 0$ and $n \rightarrow \infty$ accordingly yields

$$
g(x)-\hat{g}(x)=\frac{1}{2} x g^{2}(x) .
$$

The above formula is true for all (complex) $x$ inside the radius of convergence of $g$ (in the Appendix we show that $\hat{g}$ has a convergence radius $\beta^{-2}$ times larger than g).

For all $x$ inside the convergence radius of $g, g(x)$ must be one of the roots of the above degree 2 polynomial, i.e.

$$
g(x)=\frac{1-\sqrt{1-2 x \hat{g}(x)}}{x} .
$$

(The other root does not satisfy $g(0)=d_{0}$.)

In the Appendix we show that the radius of convergence of $\hat{g}$ is greater than 1 and that inside the unit circle of the complex plane $1-2 z \hat{g}(z)$ has only one zero, which is in fact some real point $R \in(4 / 5,1)$. It follows that $R$ is the radius of convergence of $g$, that $h$ is defined on $(0 ; R)$ and that $h\left(R^{-}\right)=0$. The other properties follow from inspection of the first coefficients $d_{k}$.

From now on $h$ is intended to be extended up to $R$. Since $h$ is bijective and has image $[0, \infty)$ and recalling that $\widetilde{a}_{n}>0$ for all $n$, it makes sense to compute $h^{-1}\left(\widetilde{a}_{n}\right)$.

Lemma 15. Let $\left\{\widetilde{a}_{n}\right\}_{n>0}$ be any positive sequence satisfying (12), not necessarily with $\widetilde{a}_{0}=0$. For $n \geq 0$ let $\lambda_{n}:=h^{-1}\left(\widetilde{a}_{n}\right) \in(0, R]$. The numbers $\lambda_{n}$ satisfy

$$
h\left(\lambda_{n+1}\right)=2\left(1-\frac{h\left(\beta^{2} \lambda_{n-1}\right)}{h\left(\lambda_{n}\right)}\right)+\frac{h\left(\beta^{4} \lambda_{n-1}\right) h\left(\beta^{2} \lambda_{n-1}\right)}{h\left(\lambda_{n}\right)}, \quad n \geq 1 .
$$

Proof. Recall that $h(x)=-\sum_{k=-1}^{\infty} d_{k} x^{k}$. We have

$$
\widetilde{a}_{n-1}^{2}=h^{2}\left(\lambda_{n-1}\right)=\sum_{i, j=-1}^{\infty} d_{i} d_{j} \lambda_{n-1}^{i} \lambda_{n-1}^{j}=\sum_{k=-2}^{\infty} \lambda_{n-1}^{k} \sum_{i=-1}^{k+1} d_{i} d_{k-i} .
$$


Using relation (13) for $k \geq-1$ and since $\beta^{2 k+2 i} \equiv 4$ if $k=-2$, we get

$$
\begin{aligned}
4 \widetilde{a}_{n-1}^{2} & =\sum_{k=-1}^{\infty} 2 d_{k} \beta^{2 k} \lambda_{n-1}^{k}+\sum_{k=-2}^{\infty} \lambda_{n-1}^{k} \sum_{i=-1}^{k+1} \beta^{2 k+2 i} d_{i} d_{k-i} \\
& =2 \sum_{k=-1}^{\infty} d_{k} \beta^{2 k} \lambda_{n-1}^{k}+\sum_{i, j=-1}^{\infty} \beta^{4 i+2 j} d_{i} d_{j} \lambda_{n-1}^{i+j} \\
& =-2 h\left(\beta^{2} \lambda_{n-1}\right)+h\left(\beta^{4} \lambda_{n-1}\right) h\left(\beta^{2} \lambda_{n-1}\right) .
\end{aligned}
$$

Substituting in equation (12) we find

$$
h\left(\lambda_{n+1}\right)=\widetilde{a}_{n+1}=2+4 \frac{\widetilde{a}_{n-1}^{2}}{\widetilde{a}_{n}}=2+\frac{-2 h\left(\beta^{2} \lambda_{n-1}\right)+h\left(\beta^{4} \lambda_{n-1}\right) h\left(\beta^{2} \lambda_{n-1}\right)}{h\left(\lambda_{n}\right)},
$$

which is equivalent to (16).

The ratio between $\lambda_{n}$ and $\beta^{2} \lambda_{n-1}$ plays a crucial role in what follows. The next lemma characterizes its logarithm.

Lemma 16. Let $\left\{\widetilde{a}_{n}\right\}_{n \geq 0}$ be any positive sequence satisfying (12), not necessarily with $\widetilde{a}_{0}=0$. For $n \geq 1$ let $\lambda_{n}^{\prime}:=\log \lambda_{n}-\log \lambda_{n-1}-\log \beta^{2}$. The sequence $\lambda_{n}^{\prime}$ can present only two behaviours:

(1) If for any $n \geq 1, \lambda_{n}^{\prime}=0$, then $\lambda_{m}^{\prime}=0$ for all $m \geq 1$.

(2) If $\lambda_{n}^{\prime} \neq 0$ for some $n \geq 1$, then there exist two constants $c, d>0$ such that for all $k \geq 0$,

$$
\left|\lambda_{n+k}^{\prime}\right| \geq c(1+d)^{k}\left|\lambda_{n}^{\prime}\right|, \quad(-1)^{k} \lambda_{n+k}^{\prime} \lambda_{n}^{\prime}>0 .
$$

Proof. If $\lambda_{n}^{\prime}=0$, then of course $\lambda_{n}=\beta^{2} \lambda_{n-1}$. Hence (16) reduces to

$$
h\left(\lambda_{n+1}\right)=h\left(\beta^{4} \lambda_{n-1}\right)=h\left(\beta^{2} \lambda_{n}\right) .
$$

Since $h$ is injective, $\lambda_{n+1}=\beta^{2} \lambda_{n}$; that is, $\lambda_{n+1}^{\prime}=0$. This proves by induction the first part of the lemma, for $k \geq n$. If the same were not true for some $k<n$, then by the second part it would have been $\lambda_{n}^{\prime} \neq 0$.

In particular note that if $\widetilde{a}_{0}=0$ (yielding $\lambda_{0}=R$ ) and $\lambda_{1}=\beta^{2} R$ (yielding $\lambda_{1}^{\prime}=0$ ), then by the first part and recurrence (12), $h\left(\beta^{4} R\right)=\widetilde{a}_{2}=2$. This will be used in the second part.

For proving the second part, let $\psi(x):=\log h\left(e^{x}\right)$ and let $\Delta_{x}(y):=h\left(x e^{y}\right) / h(x)$.

The function $\psi$ is defined on $(-\infty, \log R)$, where it is analytic, strictly decreasing, concave and invertible. $\psi$ has one oblique and one vertical asymptote, $\psi(x)+x \uparrow 0$ for $x \downarrow-\infty$ and $\psi(x) \sim \frac{1}{2} \log (\log R-x)$ for $x \uparrow \log R$, so that the image of $\psi$ is the whole $\mathbb{R}$. Moreover $\psi^{\prime}<-1$ on all the domains.

$\Delta_{x}(y)$ on the other hand is defined for $x \in(0 ; R)$ and $y \in(-\infty ; \log R-\log x)$, and this last interval always contains a neighbourhood of $0 . \Delta_{x}(\cdot)$ is analytic and decreasing on all its domains. Moreover we have

$$
\begin{aligned}
& \Delta_{x}(0)=1 \text {, } \\
& \Delta_{x}(y)>e^{-y}>1, \quad y<0, \\
& \Delta_{x}(y)<e^{-y}<1, \quad y>0 \text {. }
\end{aligned}
$$

In fact,

$$
\Delta_{x}(y)=h\left(x e^{y}\right) / h(x)=\exp (\psi(\log x+y)-\psi(\log x))=\exp \left(\psi^{\prime}(\xi) y\right)
$$


and $\psi^{\prime}(\xi)<-1$. By virtue of (16) we can write

$$
\begin{aligned}
\Delta_{\beta^{2} \lambda_{n}} & \left(\lambda_{n+1}^{\prime}\right)=\frac{h\left(\lambda_{n+1}\right)}{h\left(\beta^{2} \lambda_{n}\right)} \\
& =\frac{2}{h\left(\beta^{4} \lambda_{n-1}\right)} \frac{h\left(\beta^{4} \lambda_{n-1}\right)}{h\left(\beta^{2} \lambda_{n}\right)}\left(1-\frac{h\left(\beta^{2} \lambda_{n-1}\right)}{h\left(\lambda_{n}\right)}\right)+\frac{h\left(\beta^{4} \lambda_{n-1}\right) h\left(\beta^{2} \lambda_{n-1}\right)}{h\left(\beta^{2} \lambda_{n}\right) h\left(\lambda_{n}\right)} \\
& =\frac{2}{h\left(\beta^{4} \lambda_{n-1}\right)} \Delta_{\beta^{2} \lambda_{n}}\left(-\lambda_{n}^{\prime}\right)\left(1-\Delta_{\lambda_{n}}\left(-\lambda_{n}^{\prime}\right)\right)+\Delta_{\beta^{2} \lambda_{n}}\left(-\lambda_{n}^{\prime}\right) \Delta_{\lambda_{n}}\left(-\lambda_{n}^{\prime}\right) \\
& =2\left[\frac{1}{2}-\frac{1}{h\left(\beta^{4} \lambda_{n-1}\right)}\right] \Delta_{\beta^{2} \lambda_{n}}\left(-\lambda_{n}^{\prime}\right) \Delta_{\lambda_{n}}\left(-\lambda_{n}^{\prime}\right)+\frac{2}{h\left(\beta^{4} \lambda_{n-1}\right)} \Delta_{\beta^{2} \lambda_{n}}\left(-\lambda_{n}^{\prime}\right) .
\end{aligned}
$$

Let

$$
K_{n}:=\frac{1}{2}-\frac{1}{h\left(\beta^{4} \lambda_{n-1}\right)} \leq \frac{1}{2} .
$$

We need to prove that $K_{n}$ is always non-negative and bounded below by a positive constant for $n$ large enough.

Since $\lambda_{n-1} \leq R, h\left(\beta^{4} \lambda_{n-1}\right) \geq h\left(\beta^{4} R\right)=2$. So $K_{n} \geq 0$ for all $n \geq 1$. For $n \geq 3$, $\widetilde{a}_{n-1} \geq 2$ by (12). Hence $\lambda_{n-1} \leq \beta^{4} R$, so

$$
K_{n} \geq \frac{1}{2}-\frac{1}{h\left(\beta^{4} h^{-1}(2)\right)}=\frac{1}{2}-\frac{1}{h\left(\beta^{8} R\right)}=: K>0 .
$$

Going back to the previous expression, if $\lambda_{n}^{\prime}>0$, since both coefficients are positive,

$$
\begin{aligned}
\Delta_{\beta^{2} \lambda_{n}}\left(\lambda_{n+1}^{\prime}\right) & =2 K_{n} \Delta_{\beta^{2} \lambda_{n}}\left(-\lambda_{n}^{\prime}\right) \Delta_{\lambda_{n}}\left(-\lambda_{n}^{\prime}\right)+\left(1-2 K_{n}\right) \Delta_{\beta^{2} \lambda_{n}}\left(-\lambda_{n}^{\prime}\right) \\
& >2 K_{n} e^{2 \lambda_{n}^{\prime}}+\left(1-2 K_{n}\right) e^{\lambda_{n}^{\prime}} \\
& =2 K_{n} e^{2 \lambda_{n}^{\prime}}+\left(1-2 K_{n}\right) e^{\lambda_{n}^{\prime}}-e^{\lambda_{n}^{\prime}}+e^{\lambda_{n}^{\prime}}-1+1 \\
& =1+\left(2 K_{n} e^{\lambda_{n}^{\prime}}+1\right)\left(e^{\lambda_{n}^{\prime}}-1\right)>1 .
\end{aligned}
$$

A posteriori we get $\lambda_{n+1}^{\prime}<0$ and so, after letting $x:=e^{\lambda_{n}^{\prime}}$,

$$
e^{-\lambda_{n+1}^{\prime}}>\Delta_{\beta^{2} \lambda_{n}}\left(\lambda_{n+1}^{\prime}\right)>2 K_{n} x^{2}+\left(1-2 K_{n}\right) x=: K_{n} x(x-1)+\theta_{n}(x) \geq \theta_{n}(x),
$$

where

$$
\theta_{n}(x):=K_{n} x^{2}+\left(1-K_{n}\right) x \geq x^{1+K_{n}}=: \widetilde{\theta}_{n}(x) .
$$

The above inequality holds for all $x \geq 1$ and follows by comparing the two functions, their derivatives and by the fact that $K_{n}<1$. In fact,

$$
\begin{gathered}
\theta_{n}(1)=1=\widetilde{\theta}_{n}(1), \quad \theta_{n}^{\prime}(1)=1+K_{n}=\widetilde{\theta}_{n}^{\prime}(1), \\
\theta_{n}^{\prime \prime}(x)=2 K_{n} \geq K_{n}+K_{n}^{2} \geq\left(K_{n}+K_{n}^{2}\right) x^{\left(K_{n}-1\right)}=\widetilde{\theta}_{n}^{\prime \prime}(x), \quad \text { for } x \geq 1 .
\end{gathered}
$$

Finally $e^{-\lambda_{n+1}^{\prime}}>\widetilde{\theta}_{n}(x)=e^{\left(1+K_{n}\right) \lambda_{n}^{\prime}}$, and hence

$$
\lambda_{n}^{\prime}>0 \Rightarrow \lambda_{n+1}^{\prime}<-\left(1+K_{n}\right) \lambda_{n}^{\prime} .
$$

On the other hand, if $\lambda_{n}^{\prime}<0$, with analogous reasoning

$$
e^{-\lambda_{n+1}^{\prime}}<\Delta_{\beta^{2} \lambda_{n}}\left(\lambda_{n+1}^{\prime}\right)<2 K_{n} x^{2}+\left(1-2 K_{n}\right) x=2 K_{n} x(x-1)+x<x=e^{\lambda_{n}^{\prime}},
$$

and hence

$$
\lambda_{n}^{\prime}<0 \quad \Rightarrow \quad \lambda_{n+1}^{\prime}>-\lambda_{n}^{\prime} .
$$


Summing things up, for any $\lambda_{n}^{\prime} \neq 0$ the sequence $\left\{\lambda_{n+k}^{\prime}\right\}_{k}$ has alternating signs. Moreover,

$$
\left|\lambda_{n+k+2}^{\prime}\right| \geq\left(1+K_{n+k}\right)\left|\lambda_{n+k}^{\prime}\right| .
$$

The second part of the lemma is now proved by induction on $k$, recalling that $K_{n} \geq K>0$ for $n \geq 3$.

We are finally able to make the main statement.

Theorem 17. A sequence $a_{n}$ satisfying (11) with $a_{n_{0}}=0$ and $a_{n_{0}+1}>0$ can present only two behaviours:

(1) If for any $m \geq 1, \lambda_{m}^{\prime}=0$, then there exists $c>0$ such that for all $n>n_{0}$, $a_{n}=2^{-n} h\left(\beta^{2\left(n-n_{0}\right)} R\right)$. In this case $a_{n} \rightarrow 0$ for $n \uparrow \infty$ and in the limit $a_{n}=C_{n_{0}} \beta^{n}+O\left(\beta^{3 n}\right)$, with $C_{n_{0}}=\beta^{2 n_{0}} / R$.

(2) If for any $m \geq 1, \lambda_{m}^{\prime} \neq 0$, then either the odd or the even terms of $a_{n}$ diverge more than exponentially and the power series $\sum_{n} a_{n} z^{n}$ has zero radius of convergence.

Proof. We directly apply Lemma 16.

In the first case, for all $n \geq 1, \lambda_{n}^{\prime} \equiv 0$. Hence $\lambda_{n}=\beta^{2 n} \lambda_{0}=\beta^{2 n} R$, by which $a_{n+n_{0}}=2^{-n_{0}-n} \widetilde{a}_{n}=2^{-n_{0}-n} h\left(\beta^{2 n} R\right)$. The limit behaviour of $a_{n}$ follows from $h(x)=x^{-1}+O(1)$ for $x \downarrow 0$.

In the second case, notice that for all $k \geq 2$,

$$
\log \widetilde{a}_{k}=\log h\left(\lambda_{k}\right)=\psi\left(\log \lambda_{k}\right)=\psi\left(\log \lambda_{k-1}+\log \beta^{2}+\lambda_{k}^{\prime}\right) \geq \psi\left(\lambda_{k}^{\prime}\right) .
$$

Let $n=m$ or $n=m+1$ so that $\lambda_{n}^{\prime}<0$, and hence $\lambda_{n+2 k}^{\prime}<0$ for all $k$ and $\lambda_{n+2 k}^{\prime} \rightarrow-\infty$ for $k \uparrow \infty$. By the properties of $\psi$, there exists $x_{0}$ such that $x \leq$ $x_{0} \Rightarrow \psi(x) \geq-x-1$. We deduce that, for $k$ large enough,

$$
\log \widetilde{a}_{n+2 k} \geq \psi\left(\lambda_{n+2 k}^{\prime}\right) \geq-\lambda_{n+2 k}^{\prime}-1 \geq c(1+d)^{2 k}\left|\lambda_{n}^{\prime}\right|-1,
$$

so the power series $\sum_{k} \widetilde{a}_{k} z^{k}$ has zero radius of convergence and the same for $\sum_{k} a_{k} z^{k}$ as well.

Proof of Theorem 13. By Theorem [17, we are in the first case iff $\lambda_{1}^{\prime}=0$; that is, if $\widetilde{a}_{1}=h\left(\beta^{2} R\right)$. In the first case $\left\{a_{n}\right\} \in H$, since $a_{n}=C_{n_{0}} \beta^{n}+O\left(\beta^{3 n}\right)$ and $\beta<1$. In the second case, of course, the sequence is not in $H$.

\section{Appendix}

7.1. Proof of Theorem 3. The first claim is an obvious consequence of identity (3).

Let us prove the existence statement. Given $X^{0} \in \mathbb{R}_{+}^{\mathbb{N}}$, consider the finite dimensional system

$$
\begin{cases}X_{0}(t)=0, & \forall t \geq 0, \\ \dot{X}_{n}(t)=k_{n-1} X_{n-1}^{2}(t)-k_{n} X_{n}(t) X_{n+1}(t), & \forall t \geq 0, \quad \forall n \in\{1,2, \ldots, N\}, \\ X_{N+1}(t)=0, & \forall t \geq 0, \\ X_{n}(0)=X_{n}^{0}, & \forall n \in\{1,2, \ldots, N\} .\end{cases}
$$

It has a unique solution: local existence and uniqueness comes from the Cauchy theorem (the vector field on the right-hand side is locally Lipschitz continuous), 
and global existence is a consequence of the bound on maximal solutions derived from

$$
\sum_{n=1}^{N} X_{n}^{2}(t)=\sum_{n=1}^{N} X_{n}^{2}(0)
$$

which is easily proved by computing $\frac{d}{d t} \sum_{n=1}^{N} X_{n}^{2}(t)$. Denote by $X^{N}$ the unique solution.

Since $X^{0} \in \mathbb{R}_{+}^{\mathbb{N}}$, the solution is positive in the sense that $X_{n}^{N}(t) \geq 0$ for every $n=1, \ldots, N$ and $t \geq 0$. This simply happens because

$$
X_{n}^{N}(t)=e^{-k_{n} \int_{0}^{t} X_{n+1}^{N}(r) d r} X_{n}^{0}+\int_{0}^{t} e^{-k_{n} \int_{s}^{t} X_{n+1}^{N}(r) d r} k_{n-1}\left(X_{n-1}^{N}(s)\right)^{2} d s .
$$

Moreover, for the solution $X^{N}$, for every $n \in\{1, \ldots, N\}$ we have

$$
\frac{d}{d t} \sum_{j=1}^{n}\left(X_{j}^{N}\right)^{2}=2 \sum_{j=1}^{n} k_{j-1}\left(X_{j-1}^{N}\right)^{2} X_{j}^{N}-k_{j}\left(X_{j}^{N}\right)^{2} X_{j+1}^{N}=-k_{n}\left(X_{n}^{N}\right)^{2} X_{n+1}^{N} .
$$

Hence, being positive, we get $\frac{d}{d t} \sum_{j=1}^{n}\left(X_{j}^{N}\right)^{2} \leq 0$, namely

$$
\sum_{j=1}^{n}\left(X_{j}^{N}(t)\right)^{2} \leq \sum_{j=1}^{n}\left(X_{j}^{0}\right)^{2} \text { for all } t \geq 0 .
$$

In particular,

$$
0 \leq X_{n}^{N}(t) \leq \sqrt{\sum_{j=1}^{n}\left(X_{j}^{0}\right)^{2}} \text { for all } t \geq 0 .
$$

On a bounded interval $[0, T]$, now consider the family $\left(X_{n}^{N}\right)_{N>n}$ for a given $n \geq 1$. The assumptions of the Ascoli-Arzelà theorem are satisfied for this family. Indeed, equi-boundedness has been proved above in (18); equi-uniform-continuity (equi-Lipschitz continuity, in fact) comes from the identity

$$
X_{n}^{N}(t)-X_{n}^{N}(s)=\int_{s}^{t}\left[k_{n-1}\left(X_{n-1}^{N}(r)\right)^{2}-k_{n} X_{n}^{N}(r) X_{n+1}^{N}(r)\right] d r
$$

and the already proved equi-boundedness (always recall that $n$ is fixed). Thus, from the Ascoli-Arzelà theorem, for every $n$ there exists a sequence $\left\{N_{k}^{(n)}\right\}_{k \in \mathbb{N}}$ such that $\left\{X_{n}^{N_{k}^{(n)}}\right\}_{k \in \mathbb{N}}$ converges uniformly to a continuous function $X_{n}$. By a diagonal procedure, one can modify the previous extraction procedure and get a single sequence $\left(N_{k}\right)_{k \in \mathbb{N}}$ such that $\left(X_{n}^{N_{k}}\right)_{k \in \mathbb{N}}$ converges uniformly to $X_{n}$. We can thus pass to the limit, as $k \rightarrow \infty$, in the equation

$$
X_{n}^{N_{k}}(t)=X_{n}^{0}+\int_{0}^{t}\left[k_{n-1}\left(X_{n-1}^{N_{k}}(r)\right)^{2}-k_{n} X_{n}^{N_{k}}(r) X_{n+1}^{N_{k}}(r)\right] d r
$$

and prove that

$$
X_{n}(t)=X_{n}^{0}+\int_{0}^{t}\left(k_{n-1} X_{n-1}^{2}(r)-k_{n} X_{n}(r) X_{n+1}(r)\right) d r .
$$

Thus the functions $X_{n}(\cdot)$ are continuously differentiable and satisfy system (1). Of course they are non-negative, being the uniform limit of non-negative functions. Continuation from an arbitrary bounded time interval to all $t \geq 0$ is classical. 
Finally, we have to prove properties (i) and (ii) of the theorem for any positive componentwise solution $X$. As for (i), we repeat the argument used above for $X^{N}$ : for every $n \geq 1$, from system (11), we have

$$
\frac{d}{d t} \sum_{j=1}^{n} X_{j}^{2}=2 \sum_{j=1}^{n} k_{j-1} X_{j-1}^{2} X_{j}-k_{j} X_{j}^{2} X_{j+1}=-k_{n} X_{n}^{2} X_{n+1} .
$$

Hence, $X$ being positive, we get $\frac{d}{d t} \sum_{j=1}^{n}\left(X_{j}^{N}\right)^{2} \leq 0$, and thus (i) is proved.

As for (ii), from (3) we have

$$
X_{n}(t) \geq X_{n}^{0} e^{-k_{n} \int_{0}^{t} X_{n+1}(r) d r}>0 .
$$

For $X_{n+1}(t)$ we use the inequality (again a consequence of (3i))

$$
X_{n+1}(t) \geq \int_{0}^{t} e^{-k_{n+1} \int_{s}^{t} X_{n+2}(r) d r} k_{n} X_{n}^{2}(s) d s
$$

and the fact that $X_{n}(t)>0$ for all $t>0$. By induction we get the result. The proof of the theorem is complete.

7.2. Proof of Theorem 4. We introduce the same finite dimensional system as above. We no longer have the positivity property, but we still have (17). Since the (global) initial energy is finite, we have

$$
\sum_{n=1}^{N}\left(X_{n}^{N}(t)\right)^{2} \leq\left|X^{0}\right|_{H}^{2}
$$

for all $t \geq 0$ and $N \geq 1$. This again implies a bound on single components:

$$
\left|X_{n}^{N}(t)\right| \leq\left|X^{0}\right|_{H}
$$

for every $t \geq 0, N \geq 1$ and $n=1, \ldots, N$. Having this bound, we proceed as above and prove, on a given $[0, T]$, the existence of a componentwise solution $X$, with $X_{n}^{N_{k}} \rightarrow X_{n}$ uniformly on $[0, T]$ as $k \rightarrow \infty$, along a diverging sequence $\left(N_{k}\right)_{k \in \mathbb{N}}$. From

$$
\sum_{n=1}^{N_{k}}\left(X_{n}^{N_{k}}(t)\right)^{2} \leq\left|X^{0}\right|_{H}^{2}
$$

one easily gets

$$
\sum_{n=1}^{\infty}\left(X_{n}(t)\right)^{2} \leq\left|X^{0}\right|_{H}^{2}
$$

first by truncating the sum up to a given value $R$ and taking the limit in $k$, and then sending $R$ to infinity. Hence in particular $X(t) \in H$ for all $t \geq 0$.

Finally, assume that $X^{0} \in H \cap \mathbb{R}_{+}^{\mathbb{N}}$ and let $X$ be any componentwise solution. From Theorem 3 (i) it satisfies

$$
\sum_{j=1}^{n}\left(X_{j}(t)\right)^{2} \leq \sum_{j=1}^{n}\left(X_{j}^{0}\right)^{2} \leq\left|X^{0}\right|_{H}^{2}
$$

for every $n \geq 1$ and $t \geq 0$. This implies that $X$ is finite energy and satisfies (5). The proof is complete. 
7.3. Estimates on $\alpha_{k, i}$. Recall the definition of $\alpha_{k, i}$ :

$$
\alpha_{k, i}= \begin{cases}\frac{1+\beta^{4}-\beta^{-1}}{1-\beta^{7}-\beta^{11}}, & k=0, \quad i=0, \\ \frac{\left(1-\beta^{2 k+2}\right)\left(1+\beta^{2 k+7}\right)}{1-\beta^{2 k+7}-\beta^{4 k+11}}, & k>0, \quad i=0, k, \\ \frac{1-\beta^{3 k+6} \cosh ((k-2 i) \log \beta)}{1-\beta^{2 k+7}-\beta^{4 k+11}}, & 0<i<k .\end{cases}
$$

We study their behaviour for $k$ large and look for bounds. Notice that the denominators are all positive for $k \geq 0$.

Lemma 18. There exists $C>0$ such that for all $k \geq 0$ and all $0 \leq i \leq k$,

$$
\left|1-\alpha_{k, i}\right| \leq C \beta^{2 k} \text {. }
$$

Proof. There are three cases.

1) When $k \geq 1$ and $i=0$ or $i=k, \alpha_{k, i}<1$. In fact,

$$
\begin{aligned}
1-\alpha_{k, i} & =\frac{-\beta^{2 k+7}-\beta^{4 k+11}+\beta^{2 k+2}-\beta^{2 k+7}+\beta^{4 k+9}}{1-\beta^{2 k+7}-\beta^{4 k+11}} \\
& =\beta^{2 k}\left(\beta^{2}-\beta^{4}\right) \frac{1+\beta^{2 k+7}}{1-\beta^{2 k+7}-\beta^{4 k+11}},
\end{aligned}
$$

which is positive. The last factor is decreasing in $k$ and is equal to $4 / 3$ when $k=1$, so for $k \geq 1$,

$$
0<\beta^{2}-\beta^{4} \leq \beta^{-2 k}\left(1-\alpha_{k, i}\right) \leq 4 / 3\left(\beta^{2}-\beta^{4}\right)=: C .
$$

2) When $k \geq 2,0<i<k$, we have $\alpha_{k, i}>1$, since, recalling that $\cosh (x) \leq e^{|x|}$,

$$
\begin{aligned}
\alpha_{k, i}-1 & =\frac{-\beta^{3 k+6} \cosh ((k-2 i) \log \beta)+\beta^{2 k+7}+\beta^{4 k+11}}{1-\beta^{2 k+7}-\beta^{4 k+11}} \\
& \geq \frac{-\beta^{3 k+6} \beta^{-|k-2 i|}+\beta^{2 k+7}+\beta^{4 k+11}}{1-\beta^{2 k+7}-\beta^{4 k+11}} \\
& \geq \frac{-\beta^{2 k+8}+\beta^{2 k+7}+\beta^{4 k+11}}{1-\beta^{2 k+7}-\beta^{4 k+11}} \\
& =\beta^{2 k+7} \frac{1-\beta+\beta^{2 k+4}}{1-\beta^{2 k+7}-\beta^{4 k+11}} .
\end{aligned}
$$

The right factor is decreasing in $k$; hence for $k \geq 2$,

$$
\beta^{-2 k}\left(\alpha_{k, i}-1\right) \geq \beta^{7}-\beta^{8}>0 .
$$

From $\cosh (x) \geq 1$ it follows that

$$
\begin{aligned}
\alpha_{k, i}-1 & \leq \frac{-\beta^{3 k+6}+\beta^{2 k+7}+\beta^{4 k+11}}{1-\beta^{2 k+7}-\beta^{4 k+11}} \\
& =\beta^{2 k+7} \frac{1-\beta^{k-1}+\beta^{2 k+4}}{1-\beta^{2 k+7}-\beta^{4 k+11}} .
\end{aligned}
$$

The right factor is increasing in $k$ for $k \geq 2$ and it tends to 1 , so

$$
0<\beta^{7}-\beta^{8} \leq \beta^{-2 k}\left(\alpha_{k, i}-1\right) \leq \beta^{7}<C .
$$

The third case, namely $k=0$, is included by adjusting $C$. 
7.4. Estimates on the convergence radius of $\hat{g}$ and $g$. By equation (15), in order to find the radius of convergence of $g$, we need to know the zeros of $\tilde{g}(z):=1-2 z \hat{g}(z)$.

Theorem 19. The complex function $\tilde{g}$ has only one root inside the unit circle centered in the origin. This root is a real number $R \in(4 / 5,1)$.

The proof is based on Rouché's theorem for holomorphic functions.

We split $\tilde{g}$ in the sum of two terms: $\tilde{g}=\tilde{g}_{A}+\tilde{g}_{B}$, where $\tilde{g}_{A}(z)=1-2 d_{0}^{\prime} z-2 d_{1}^{\prime} z^{2}$, and $\tilde{g}_{B}(z)=-2 z \sum_{k=2}^{\infty} d_{k}^{\prime} z^{k}$. Rouché's theorem tells us that if $\left|\tilde{g}_{A}\right|>\left|\tilde{g}_{B}\right|$ on the contour of some compact set of $\mathbb{C}$, then $\tilde{g}_{A}$ and $\tilde{g}$ have the same number of zeros inside the compact.

We will prove that $\left|\tilde{g}_{A}\right|>\left|\tilde{g}_{B}\right|$ on the border of two circles with centre in the origin and radii 1 and $4 / 5$.

In the following we first give a function $G$ that is an upper bound for $g$. Then we study separately the maximum of $\left|\tilde{g}_{B}\right|$ and the minimum of $\left|\tilde{g}_{A}\right|$ on the two circles. After that we prove the theorem.

An upper bound for $g$. Let

$$
M:=\frac{1}{2} \max _{k, i} \alpha_{k, i}=\alpha_{2,1} / 2 .
$$

(It follows from the fact that $\max _{i} \alpha_{k, i}=\alpha_{k,[k / 2]}$ and that $\alpha_{k,[k / 2]}$ is decreasing in $k$.)

We introduce another auxiliary sequence $\left\{D_{k}\right\}_{k}$ :

$$
\left\{\begin{array}{l}
D_{0}=d_{0} \\
D_{1}=d_{1} \\
D_{k+1}=M \sum_{i=0}^{k} D_{i} D_{k-i}, \quad k \geq 1 .
\end{array}\right.
$$

Lemma 20. Let $G(z):=\sum_{k=0}^{\infty} D_{k} z^{k}$. The radius of convergence of $g$ is larger than the radius of convergence of $G$, which is larger than $\beta^{2}$.

Proof. Thanks to (14), by induction $0 \leq d_{k} \leq D_{k}$ for all $k$.

By adding the third part of (20) we find that

$$
G(z)-d_{0}-d_{1} z=\sum_{k \geq 1} D_{k+1} z^{k+1}=M z \sum_{k \geq 1} \sum_{i=0}^{k} D_{i} z^{i} D_{k-i} z^{k-i}=M z G(z)^{2}-M d_{0}^{2} z .
$$

Hence for $z \in \mathbb{C}$ inside the convergence radius of the series,

$$
G(z)=\frac{1 \pm \sqrt{1-4 M z\left(d_{0}+\left(d_{1}-M d_{0}^{2}\right) z\right)}}{2 M z} .
$$

The right sign is '-' for all $z$ since $G(0)=d_{0}$ and $G$ is continuous. After some algebraic manipulations we write

$$
G(z)=\frac{1-\sqrt{1-4 M d_{0} z+4 A M^{2} d_{0}^{2} z^{2}}}{2 M z},
$$

where $A=1-\alpha_{0,0} / 2 M$.

The radius of convergence of $G$ is the distance from the origin of the nearest zero of the radicand. The roots of the latter are both real and positive:

$$
z_{1,2}=\frac{1 \pm \sqrt{\alpha_{0,0} / 2 M}}{2 A M d_{0}} .
$$


The smallest one is the convergence radius of $G$, and one can verify that

$$
z_{1}=\frac{1-\sqrt{\alpha_{0,0} / \alpha_{2,1}}}{\left(\alpha_{2,1}-\alpha_{0,0}\right) d_{0}}>\beta^{2} .
$$

This completes the proof.

\section{On the convergence of $\hat{g}, \tilde{g}$ and the maximum of $\tilde{g}_{B}$.}

Lemma 21. The radius of convergence of $\hat{g}$ and hence $\tilde{g}$ is greater than $1 . \tilde{g}_{B}$ on the circumferences of radii 1 and $4 / 5$ is bounded by

$$
\begin{aligned}
\left|\tilde{g}_{B}\left(e^{i \theta}\right)\right| & \leq 0.062, & \forall \theta \in[0,2 \pi], \\
\left|\tilde{g}_{B}\left(4 / 5 e^{i \theta}\right)\right| & \leq 0.031, & \forall \theta \in[0,2 \pi] .
\end{aligned}
$$

Proof. Let $z \in \mathbb{C}$. Then:

$$
\left|\tilde{g}_{B}(z)\right| \leq 2|z| \sum_{k=2}^{\infty}\left|d_{k}^{\prime}\right||z|^{k}=2 \sum_{k=2}^{\infty}\left|d_{k}^{\prime}\right||z|^{k+1} .
$$

By the estimates on $\alpha_{k, i}$, we have $\left|1-\alpha_{k, i}\right|<C \beta^{2 k}$; we also need some lower bound and, for $k \geq 1, \alpha_{k, i} \geq \alpha_{1,0}$. Then for all $k \geq 1$,

$$
\begin{aligned}
\left|d_{k+1}^{\prime}\right|=\mid \frac{1}{2} \sum_{i=0}^{k}\left(\alpha_{k, i}-1\right) & d_{i} d_{k-i} \mid \leq \frac{1}{2} C \beta^{2 k} \sum_{i=0}^{k} d_{i} d_{k-i} \\
\leq & \frac{C \beta^{2 k}}{\alpha_{1,0}} \frac{1}{2} \sum_{i=0}^{k} \alpha_{k, i} d_{i} d_{k-i}=\frac{C \beta^{2 k}}{\alpha_{1,0}} d_{k+1} \leq \frac{C \beta^{2 k}}{\alpha_{1,0}} D_{k+1} .
\end{aligned}
$$

Thanks to (22), this proves that the radius of convergence of $\hat{g}$ is greater than 1 .

Putting together the last two upper bounds we get

$$
\begin{aligned}
\left|\tilde{g}_{B}(z)\right| \leq 2 \sum_{k=2}^{\infty} \frac{C \beta^{2 k-2}}{\alpha_{1,0}} D_{k}|z|^{k+1}=\frac{C \beta^{-5}}{\alpha_{1,0}} \sum_{k=2}^{\infty} \beta^{2 k}|z|^{k+1} D_{k} \\
=\frac{C \beta^{-5}|z|}{\alpha_{1,0}}\left(G\left(\beta^{2}|z|\right)-D_{0}-D_{1} \beta^{2}|z|\right) .
\end{aligned}
$$

The value of $G\left(\beta^{2}|z|\right)$ can be computed thanks to (21), and the bounds one gets are those in the lemma.

\section{On the minimum of $\tilde{g}_{A}$.}

Lemma 22. The maximum and the minimum of $\left|\tilde{g}_{A}(z)\right|$ on the circumferences with centre in the origin and radii 1 and $4 / 5$ are on the following points:

$$
\begin{aligned}
\tilde{g}_{A}(-1) & \approx 3.170, & \tilde{g}_{A}(1) & \approx-0.092, \\
\tilde{g}_{A}(-4 / 5) & \approx 2.650, & \tilde{g}_{A}(4 / 5) & \approx 0.040 .
\end{aligned}
$$


Proof. We study $\left|\tilde{g}_{A}\left(\rho e^{i \theta}\right)\right|$. Recall that $\tilde{g}_{A}(z)=1-2 d_{0}^{\prime} z-2 d_{1}^{\prime} z^{2}=\sum_{k=0}^{2} c_{k} z^{k}$ is a polynomial with real coefficients. Therefore,

$$
\begin{array}{r}
\left|\tilde{g}_{A}\left(\rho e^{i \theta}\right)\right|^{2}=\tilde{g}_{A}\left(\rho e^{i \theta}\right) \overline{\tilde{g}_{A}\left(\rho e^{i \theta}\right)}=\tilde{g}_{A}\left(\rho e^{i \theta}\right) \tilde{g}_{A}\left(\rho e^{-i \theta}\right)=\sum_{j, k=0}^{2} c_{j} c_{k} \rho^{j+k} e^{i \theta(j-k)} \\
=\sum_{j=0}^{2} c_{j}^{2} \rho^{2 j}+2\left(c_{0} c_{1} \rho+c_{1} c_{2} \rho^{3}\right) \cos (\theta)+2 c_{0} c_{2} \rho^{2} \cos (2 \theta) .
\end{array}
$$

By setting $\frac{\partial}{\partial \theta}\left|\tilde{g}_{A}\left(\rho e^{i \theta}\right)\right|^{2}=0$ we find that

$$
\begin{aligned}
0 & =\left(c_{0} c_{1}+c_{1} c_{2} \rho^{2}\right) \sin (\theta)+2 c_{0} c_{2} \rho \sin (2 \theta) \\
& =\left(c_{0} c_{1}+c_{1} c_{2} \rho^{2}+4 c_{0} c_{2} \rho \cos (\theta)\right) \sin (\theta) .
\end{aligned}
$$

The first factor is never 0 because $\left|c_{0} c_{1}+c_{1} c_{2} \rho^{2}\right|-\left|4 c_{0} c_{2} \rho\right|>0$. In fact,

$$
\begin{gathered}
\left|c_{0} c_{1}+c_{1} c_{2} \rho^{2}\right|-\mid \\
=2 c_{0} c_{2} \rho\left|=2 d_{0}^{\prime}\right| 1-2 d_{1}^{\prime} \rho^{2}|-8| d_{1}^{\prime} \mid \rho \\
=2 d_{0}\left(1+\left(1-\alpha_{0,0}\right) d_{0}^{2} \rho^{2}\right)-4\left(1-\alpha_{0,0}\right) d_{0}^{2} \rho \\
>2 d_{0}\left[1+\left(1-\alpha_{0,0}\right) d_{0}^{2} \rho^{2}-2\left(1-\alpha_{0,0}\right) d_{0} \rho\right] \\
\left.\quad=2 d_{0}^{2} \rho^{2}-2\left(1-\alpha_{0,0}\right) d_{0} \rho\right] \\
\left.\quad\left(1-\alpha_{0,0}\right) d_{0} \rho\right)^{2} \geq 0 .
\end{gathered}
$$

So the maximum and the minimum of $\left|\tilde{g}_{A}(z)\right|$ on the circumference with centre in the origin and radius $\rho$ are on the real points $\pm \rho$.

Setting $\rho=1$ and $\rho=4 / 5$ and computing values, we get the thesis.

Proof of Theorem 19. Direct computation shows that $\tilde{g}_{A}$ has only one root inside the unit circle, in some real point in $(4 / 5,1)$. By Rouché's theorem and the bounds of Lemmas 21 and 22 above, $\tilde{g}$ has only one root inside the circular crown with centre in the origin and radii $4 / 5$ and 1 . This root is a real number $R \in(4 / 5,1)$, since the same estimates state that (by continuity) $\tilde{g}$ must be zero for some real number in the interval:

$$
\tilde{g}(1)=\tilde{g}_{A}(1)+\tilde{g}_{B}(1)<0<\tilde{g}_{A}(4 / 5)+\tilde{g}_{B}(4 / 5)=\tilde{g}(4 / 5) .
$$

\section{REFERENCES}

[1] D. Barbato, M. Barsanti, H. Bessaih, F. Flandoli, Some rigorous results on a stochastic GOY model, J. Stat. Phys. 125 (2006), no. 3, 677-716. MR.2281460(2009c:76034)

[2] R. Benzi, B. Levant, I. Procaccia, E.S. Titi, Statistical properties of nonlinear shell models of turbulence from linear advection model: rigorous results, Nonlinearity 20 (2007), no. 6, 1431-1441. MR2327131 (2008m:76065)

[3] L. Biferale, Shell Models of Energy Cascade in Turbulence, Annu. Rev. Fluid. Mech. 35 (2003), 441-468. MR.1967019 (2004b:76074)

[4] A. Cheskidov, Blow-up in finite time for the dyadic model of the Navier-Stokes equations, Trans. Amer. Math. Soc. 360 (2008), no. 10, 5101-5120. MR2415066 (2010a:35180)

[5] A. Cheskidov, S. Friedlander, N. Pavlovic, Inviscid dyadic model of turbulence: The fixed point and Onsager's conjecture, J. Math. Phys. 48 (2007), no. 6, 065503, 16 pp. MR2337019 (2008k:76012)

[6] A. Cheskidov, S. Friedlander, N. Pavlovic, An inviscid dyadic model of turbulence: The global attractor, arXiv:math.AP/0610815.

[7] P. Constantin, B. Levant, E. S. Titi, Regularity of inviscid shell models of turbulence, Phys. Review E (3) 75 (2007), no. 1, 016304, 1-10. MR2324676 (2008e:76085) 
[8] S. Friedlander, N. Pavlovic, Blowup in a three-dimensional vector model for the Euler equations, Comm. Pure Appl. Math. 57 (2004), no. 6, 705-725. MR.2038114 (2005c:35241)

[9] U. Frisch, Turbulence, Cambridge University Press, Cambridge (1995). MR.1428905 (98e:76002)

[10] G. Gallavotti, Foundations of Fluid Dynamics, Texts and Monographs in Physics, SpringerVerlag, Berlin, 2002. MR1872661 (2003e:76002)

[11] N. H. Katz, N. Pavlovic, Finite time blow-up for a dyadic model of the Euler equations, Trans. Amer. Math. Soc. 357 (2005), no. 2, 695-708. MR2095627(2005h:35284)

[12] A. Kiselev, A. Zlatoš, On discrete models of the Euler equation, IMRN 38 (2005), no. 38, 2315-2339. MR2180809 (2007e:35229)

[13] A. N. Kolmogorov, The local structure of turbulence in incompressible viscous fluids at very large Reynolds numbers, Dokl. Akad. Nauk. SSSR 30 (1941), 301-305. MR0004146 (2:327d)

[14] F. Waleffe, On some dyadic models of the Euler equations, Proc. Amer. Math. Soc. 134 (2006), 2913-2922. MR2231615 (2007g:35222)

Dipartimento di Matematica Pura e Applicata, Università degli Studi di Padova, Via Treste 63, 35121 Padova, Italy

Department of Applied Mathematics, University of Pisa, Via Buonarroti 1, 56127 Pisa, Italy ITALY

Department of Mathematics, University of Parma, Parco Scienze 53A, 43124 Parma, 